\title{
Hiperglicemia e Doença Cardiovascular Podem Coexistir
}

F

XPRESSÕES COMO "diabetes é uma doença vascular, o paciente com diabetes é um coronariopata em potencial, pacientes com diabetes têm o mesmo risco em desenvolver um evento cardiovascular quando comparados com não-diabéticos que já tiveram um infarto do miocárdio" têm sido comumente utilizadas e cada vez mais difundidas. Realmente tem sido recomendado que os pacientes com diabetes tipo 2 sejam considerados portadores de doença coronariana e como tal devam ser tratados, incluindo, por exemplo, metas mais rígidas de controle de colesterol e suas frações e dos níveis pressóricos (prevenção secundária).

A causa maior das internações hospitalares de pacientes com diabetes é em razão da doença cardiovascular. A elevada taxa de morbidade e mortalidade nesses pacientes é, sem dúvida, também conseqüência de manifestações de doença cardiovascular. $\mathrm{O}$ infarto do miocárdio e o acidente vascular cerebral são as principais causas de morte dos pacientes com diabetes (1-4). Especialistas em cardiologia têm cada vez mais se envolvido no tratamento do diabetes. Por outro lado, endocrinologistas têm também se envolvido mais e mais em estudos sobre a patogênese da aterosclerose, tentando compreender melhor as relações entre, por exemplo, resistência à insulina, adipoquinas, inflamação e desenvolvimento da doença coronariana.

Sociedades médicas, como a American Diabetes Association, têm lançado diretrizes para o diagnóstico de doença coronariana em pacientes portadores de diabetes $(5,6)$. Em excelente revisão, Young e col. (7) discutem quais pacientes com diabetes devem ser avaliados. É óbvio que possam existir barreiras econômicas. Um estudo recente realizado no Centro de Diabetes da Universidade Federal de São Paulo (8) demonstrou que a avaliação cardiovascular em um centro universitário está aquém do mínimo necessário. Apesar disso, a maioria dos endocrinologistas, quando existe disponibilidade para tal, tem-se empenhado mais nesse tipo de avaliação. A solicitação do eletrocardiograma de esforço, do ecodopplercardiograma, da cintilografia do miocárdio, da tomografia computadorizada de coronárias e de outros testes cardiológicos tem-se tornado mais freqüente no consultório do endocrinologista.

Esse fato demonstra uma mudança de paradigma e expressa que absolutamente não devemos ser "glucocêntricos" ao extremo, mas entender que o desfecho final de qualquer tratamento para diabetes deve ter como objetivo a prevenção da doença cardiovascular. Contudo, e supreendentemente, nenhum agente oral demonstrou, até o momento, atuar na prevenção de eventos cardiovasculares. Atualmente, discute-se mais a segurança de tais agentes em termos de doença cardiovascular do que propriamente a proteção.

A importância do tema diabetes e doença cardiovascular pode ser demonstrada pela recente edição dos Arquivos Brasileiros de Endocrinologia e Metabolismo, órgão oficial da Sociedade Brasileira de Endocrinologia e Metabolismo com um número especial dedicado ao tema (9).

\section{editorial}

\section{Antonio Roberto Chacra \\ Marcelo Alves Alvarenga}

Professor titular, Chefe da

Disciplina de Endocrinologia do

Departamento de Medicina da

Escola Paulista de Medicina da Universidade Federal de São Paulo (Unifesp/EPM), São Paulo, SP, Brasil, (ARC).

Médico do Centro de Pesquisa Clínica em Diabetes da Unifesp/ EPM, São Paulo, SP, Brasil (MAA). 
Contudo, qual é a exata relação entre a hiperglicemia e macroangiopatia? A hiperglicemia é sinônimo de diabetes? Qual é a importância da hiperglicemia pósprandial no desenvolvimento da aterosclerose? $\mathrm{O}$ controle rigoroso da hiperglicemia realmente previne o aparecimento e a progressão da aterosclerose? A questão não tem sido respondida de maneira conclusiva, principalmente quando consideramos outras variáveis como peso, idade do paciente, duração do diabetes, grau de resistência à insulina, presença de outras comorbidades como dislipidemia, hipertensão, além de outros fatores de risco como tabagismo, sedentarismo e estresse. Por outro lado, também não parece haver dúvidas de que o paciente com diabetes tem risco maior em desenvolver um infarto do miocárdio do que um indivíduo sem diabetes, e que a aterosclerose na população diabética é mais séria, mais freqüente e ocorre mais precocemente quando comparada com uma população sem diabetes. Esse fato é decorrente da hiperglicemia ou de algum outro fator, como resistência insulínica presente ou associada ao diabetes?

A relação entre hiperglicemia de estresse, diabetes e evento coronariano agudo pode ser mais complexa. Denomina-se hiperglicemia de estresse a elevação da glicose sanguínea durante a admissão hospitalar de pacientes com infarto agudo do miocárdio sem histórico prévio de diabetes. O presente número dos Arquivos Brasileiros de Endocrinologia e Metabologia (ABEM) apresenta os resultados de uma pesquisa realizada no Instituto do Coração da Faculdade de Medicina da Universidade de São Paulo que avalia a prevalência, talvez mais bem denominada de freqüência da hiperglicemia de estresse e diabetes, em pacientes com infarto agudo do miocárdio (10). Os dados são interessantes no sentido que demonstram uma elevada taxa de alterações glicêmicas nesses pacientes $(37,4 \%)$. A glicemia alta torna pior o prognóstico desses pacientes? Lerario e col. mencionam no referido artigo que a hiperglicemia de estresse pode estar associada a pior desfecho com significante aumento da mortalidade hospitalar. Realmente Malmberg e col. (11) demonstraram, no bastante conhecido estudo DIGAMI, que a correção da glicemia por meio de insulinização intensiva no período intra-hospitalar reduziu significantemente a mortalidade intra-hospitalar, assim como após um ano de posterior acompanhamento. Os autores relatam também uma freqüência menor de intervenção cirúrgica nesses pacientes. Decorrência de maior gravidade da função cardíaca ou maior cautela em intervir em pacientes com hiperglicemia.

Em conclusão, é interessante notar que a presente pesquisa foi realizada em uma instituição altamente especializada em cardiologia, pesquisa esta que foi dirigida por endocrinologistas de renome em um setor do Instituto do Coração (InCor), denominado Núcleo de Diabetes e Coração, mostrando mais uma vez o caráter sistêmico da moléstia diabetes e a necessidade, sem dúvida, de maior integração entre a cardiologia e a endocrinologia.

\section{REFERÊNCIAS}

1. Morrish NJ, Wang SL, Stevens LK, Fuller JH, Keen H. Mortality and causes of death in the WHO Multinational Study of Vascular Disease in Diabetes. Diabetologia. 2001;44(Suppl 2):S14-21.

2. Meigs JB. Epidemiology of cardiovascular complications in type 2 diabetes. Acta Diabetol 2003;40:S358-61.

3. Kannel WB, McGee DL. Diabetes and cardiovascular disease: the Framingham Study 1999. JAMA. 241:2035-8.

4. Lessa I. Relative mortality trends for diabetes mellitus in the Brazilian state capitals, (1950-1985). Bol Oficina Sanit Panam. 1992;113(3):212-7.

5. American Diabetes Association: Consensus development conference on the diagnosis of coronary heart disease in people with diabetes: 10-11 February 1998, Miami, Florida. Diabetes Care. 1998;21:1551-9.

6. Standards of Medical Care in Diabetes - 2006, American Diabetes Association. Diabetes Care. 2006:29:S4-42.

7. Young LH, Jose $P$, Chvun D. Diagnosis of CAD in patients with diabetes: who to evaluate? Curr Diab Rep. 2003;3:19-27.

8. Alvarenga MA, Oliveira CSV, Chacra AR, Reis AF. Avaliação Cardiovascular de Pacientes Diabéticos em Ambulatório Universitário. Arq Bras Endocrinol Metab. 2007;51/52:281-4.

9. Schraan BD, Reis AF. Diabetes Mellittus e Doença Cardiovascular. Ed especial. Arq Bras Endocrinol Metabol. 2007:149-362.

10. Lerario AC, Coretti FMLM, Oliveira SF, Betti RTB, Bastos MSCB, Ferri LAF, et al. Avaliação da prevalência do diabetes e da hiperglicemia de estresse no infarto agudo do miocárdio. Arq Bras Endocrinol Metab. 2008; 52(3): 465-72.

11. Malmberg K, Ryden L, Efendic S, Herlitz J, Niol P, Walstrom A, et al. Randomized trial of insulin glucose infusion followed by subcutaneous insulin treatment in diabetic patients with acute myocardial infarction (Digami Study): effects on mortality al 1 year. J Am Coll Cardiol. 1995;26:57-65.

Endereço para correspondência:

Antonio Roberto Chacra

Alameda Franca, 910

01422-001 São Paulo, SP

E-mail: chacra@unifesp.br 